\title{
Optimal management of postoperative parenchymal air leaks
}

\author{
Daniel G. French, Madelaine Plourde, Harry Henteleff, Aneil Mujoomdar, Drew Bethune
}

Division of Thoracic Surgery, Department of Surgery, Queen Elizabeth II Hospital - Victoria Campus, Dalhousie University, Halifax, NS, Canada Contributions: (I) Conception and design: DG French, M Plourde; (II) Administrative support: None; (III) Provision of study materials or patients: None; (IV) Collection and assembly of data: None; (V) Data analysis and interpretation: None; (VI) Manuscript writing: All authors; (VII) Final approval of manuscript: All authors.

Correspondence to: Daniel G. French, MD, FRCSC. Assistant Professor of Surgery, Division of Thoracic Surgery, Department of Surgery, Queen Elizabeth II Hospital - Victoria Campus, Dalhousie University, 1276 South Park Street, Halifax, NS, Canada. Email: danielg.french@nshealth.ca.

\begin{abstract}
Air leaks are the most common complication after pulmonary resection. Enhanced recovery after surgery (ERAS) programs must be designed to manage parenchymal air leaks. ERAS programs should consider two components when creating protocols for air leaks: assessment and management. Accurate assessment of air leaks using traditional analogues devices, newer digital drainage systems, portable devices and chest X-rays (CXR) are reviewed. Published data suggests that digital drainage systems result in a more confident assessment of air leaks. The literature regarding the management of postoperative air leaks, including the number of chest tubes, the role of applied external suction, invasive maneuvers and discharge with a portable device is reviewed. The key findings are that a single chest drain is adequate in the majority of cases to manage an air leak, the use of applied external suction is unlikely to prevent or prolong an air leak, autologous blood patch pleurodesis may potentially shorten postoperative air leaks and there is sufficient data to support that patients can safely be discharged with a portable drainage system. There is also literature to support the design of protocols for management of postoperative air leaks. Standardization of postoperative care through ERAS programs will allow for the design of larger RCTs to better understand some of the controversies around the management of postoperative air leaks.
\end{abstract}

Keywords: Enhanced recovery after surgery (ERAS); chest drain protocol; chest tube management

Submitted May 02, 2018. Accepted for publication Oct 05, 2018.

doi: $10.21037 /$ jtd.2018.10.05

View this article at: http://dx.doi.org/10.21037/jtd.2018.10.05

Post-lung resection air leaks remain one of the most common complications resulting in delay of hospital discharge (1). They result from an alveolar-pleural fistula, and in most instances, will heal spontaneously. Prolonged air leaks (PAL) have been defined as lasting more than 5 days after surgery (2). Despite multiple advances in lung resection, including the use of stapling devices, sealants and approaches without fissure dissection, thoracic surgeons continue to be plagued by air leaks. Over $50 \%$ of patients undergoing lung resection will have an air leak within the first 24 hours after surgery (2-4) and up to $15 \%$ of patients will have a PAL (5).

Enhanced recovery after surgery (ERAS) programs must be designed to deal with air leaks in a systemic, evidencebased manner. Management of air leaks spans from the preoperative assessment to predict patients at high risk of PAL, intraoperative maneuvers to prevent parenchymal air leaks and postoperative management to reduce the duration of an air leak. This manuscript will focus on the two components of postoperative management of PAL: (I) accurate assessment of the air leak, and (II) management of a true alveolar-pleural fistula.

\section{Air leak assessment}

The accurate measurement of an air leak following lung resection has come to the forefront of ERAS programs. Traditional analogue devices only allow for a subjective static assessment of air leaks. Digital devices have allowed more objective measurements of air leaks by measuring 
and recording the continuous airflow as volume per time (i.e., $\mathrm{mL} / \mathrm{min}$ ). The role of routine chest $\mathrm{X}$-rays (CXR) has also been recently reconsidered. And, patients are being discharged with portable devices and therefore requiring outpatient assessment of air leaks. It is important to note the assessment discussed below is limited to an air leak from alveolar-pleural fistula and not a broncho-pleural fistula (BPF), which requires a more complex assessment and management plan.

\section{Analogue devices}

Initial chest drainage devices were based on a three-bottle system. Current analogue drainage systems comprise of wet or dry suction controls. The dry suction system allows for higher suction pressures. The most common devices used today are the Pleur-evac (Teleflex Incorporated, USA) and Atrium (Maquet Getinge Group, Germany) systems. These devices consist of a collection chamber for fluid and a water seal chamber for measurement of air leak. An air leak is measured by bubbling in the water seal chamber. Grading of the air leak is done by recording the numbered column the bubbles reach when the patient coughs or exhales; the higher the number reached the larger the air leak. A few classifications have attempted to quantify air leaks. The most commonly cited is the Robert David Cerfolio Classification System (6). This classification lists grades 1 through 4 with grade 1 air leaks being present with forced expiration/cough, grade 2 with expiration, grade 3 with inspiration and grade 4 with continuous bubbling during both inspiration and expiration. Viewing these bubbles in the water seal chamber is subjective and allows for interobserver variability $(7,8)$. This can necessitate uncertainty regarding the presence or absence of a small air leak and lead to chest tube clamping trials. Clamping trials delay discharge in patients without a true air leak and carry the risk of a symptomatic pneumothorax and accumulation of subcutaneous air in patients with a true air leak.

\section{Digital devices}

Digital measurements of air leaks are now available with Thopaz (Medela Healthcare, Baar, Switzerland) and Atmos (MedizinTechnik, Germany) devices. These devices allow the continuous measure of airflow and allow the recording of air leaks which can be visualized as a graph over 12-48 hours. This minimizes the subjective assessment of air bubbles and decreases interobserver variability $(7,8)$. The potential benefits from the more objective measurements offered by digital drainage devices are earlier chest tube removal, fewer clamping trials and prediction of PAL. Table 1 provides a summary of multiple studies that have been conducted with the primary outcomes of length of stay (LOS) and chest tube duration. There are currently ten randomized trials that have compared digital and analog chest drainage devices. Six trials concluded that the digital devices resulted in shorter chest tube duration and shorter LOS (9-14). One study showed shorter chest tube duration with no significant difference in LOS (15). Three trials did not find a significant difference in either chest tube duration or LOS (16-18). Two RCTs determined that digital devices resulted in fewer clamping trials $(17,18)$.

Potential explanations for these different results are the lack of consensus on the ideal amount of peak airflow and the amount of chest tube drainage prior to chest tube removal. To date there has not been consensus on the minimal airflow required prior to chest tube removal. Airflow spikes from 0 to $40 \mathrm{~mL} / \mathrm{min}$ over various time intervals (8-12 hours) have been used as thresholds prior to chest tube removal. In addition to air leaks, fluid output is an additional criterion to be considered prior to chest tube removal. There is also no consensus on the ideal amount of output over 24 hours with accepted ranges from 200 to $450 \mathrm{~mL}$ per 24 hours (9-14,16,17). Furthermore, chest tubes are not necessarily removed as soon as the air leak has resolved. Chest tubes are typically removed during the day following assessment of the device during morning rounds. Regular assessment of air leaks with chest tube removal as soon as criteria are met would be required in order to truly benefit from the objective continuous monitoring of digital devices.

Protocols developed based on the results of digital devices will result in more streamlined chest tube management. The objective measure of air leaks will allow nursing staff to determine when an air leak has resolved. This could then result in earlier chest tube removal and discharge from hospital. A future direction with the use of digital devices is the prediction of patients who will have a PAL. The prediction of PAL could identify which patients would benefit from earlier discharge from hospital with a portable device.

\section{Portable devices}

The most commonly used portable devices are a Heimlich valve and a Pneumostat (Atrium, Hudson, USA) (19). Heimlich valves allow air to escape but prevent re-entry via 
Table 1 Results of ten clinical trials comparing digital devices to traditional chest tube drainage systems

\begin{tabular}{|c|c|c|c|}
\hline Publication & Number of patients & Results & Conclusion \\
\hline Filosso et al. (11) & 31 & Shorter LOS $(P=0.00001)$; lower cost $(P=0.00005)$ & $\begin{array}{c}\text { Favors digita } \\
\text { devices }\end{array}$ \\
\hline Bertolaccini et al. (12) & 98 & $\begin{array}{l}\text { Shorter LOS ( } 6.5 \text { vs. } 7.1 \text { days) }(P=0.09) \text {; shorter chest tube duration } \\
\text { ( } 5.5 \text { vs. } 6.1 \text { days) }(P=0.06)\end{array}$ & $\begin{array}{c}\text { Favors digita } \\
\text { devices }\end{array}$ \\
\hline Pompili et al. (13) & 381 & $\begin{array}{l}\text { Shorter LOS ( } 4.6 \text { vs. } 5.6 \text { days) }(P<0.0001) \text {; shorter chest tube duration } \\
\text { ( } 3.6 \text { vs. } 4.7 \text { days) }(P<0.0001)\end{array}$ & $\begin{array}{c}\text { Favors digital } \\
\text { devices }\end{array}$ \\
\hline Filosso et al. (14) & 80 & $\begin{array}{l}\text { Shorter LOS by }(7 \text { vs. } 8 \text { days })(P<0.05) \text {; shorter chest tube duration } \\
\text { ( } 3 \text { vs. } 5 \text { days })(P<0.05)\end{array}$ & $\begin{array}{l}\text { Favors digital } \\
\text { devices }\end{array}$ \\
\hline Lijkendijk et al. (16) & 105 & $\begin{array}{l}\text { No difference in LOS ( } 4 \text { vs. } 5 \text { days) }(\mathrm{HR}=0.91 ; \mathrm{P}=0.65) \text {; no difference } \\
\text { in chest tube duration ( } 42 \text { vs. } 46.5 \text { hours) }(\mathrm{HR}=0.84 ; \mathrm{P}=0.4)\end{array}$ & No difference \\
\hline \multirow[t]{3}{*}{ Gilbert et al. (17) } & $\begin{array}{l}176 \text { patients stratified } \\
\text { by air leak status }\end{array}$ & $\begin{array}{l}\text { Air leak absent; no difference in LOS ( } 4.0 \text { vs. } 4.0 \text { days) }(P=0.09) \text {; } \\
\text { no difference in chest tube duration ( } 3.0 \text { vs. } 2.9 \text { days) }(P=0.05)\end{array}$ & No difference \\
\hline & & $\begin{array}{l}\text { Air leak present; no difference in LOS ( } 6.0 \text { vs. } 6.0 \text { days) }(P=0.36) \text {; } \\
\text { no difference in chest tube duration ( } 5.6 \text { vs. } 4.9 \text { days) }(P=0.11)\end{array}$ & \\
\hline & & Overall decreased number of chest tube clamping trials $(P=0.01)(9)$ & \\
\hline Plourde et al. (18) & 215 & $\begin{array}{l}\text { No difference in LOS ( } 4 \text { vs. } 5 \text { days) }(\mathrm{P}=0.47) \text {; no difference in chest } \\
\text { tube duration ( } 3 \text { vs. } 3 \text { days) }(\mathrm{P}=0.2) \text {; decreased number of chest tube } \\
\text { clamping trials }(\mathrm{P}<0.0001)\end{array}$ & No difference \\
\hline
\end{tabular}

LOS, length of stay.

rubber one-way valve. A Pneumostat has a one-way valve and a collection chamber to capture effluent.

To assess for an air leak in a Heimlich valve the device is submerged in water and the patient is asked to generate positive intrathoracic pressure (i.e., cough or Valsalva maneuver). An alveolar-pleural fistula is present if bubbles appear in the water (20). A Pneumostat has a small chamber where a water or saline can be instilled. Again, the patient is asked to generate positive intrathoracic pressure. If bubbles appear in the liquid, an air leak is present. Many patients can be instructed to check for air leaks at home, and return to hospital on resolution of the air leak for removal of the chest drain (21).

\section{Role of routine CXR}

CXR are commonly ordered in the postoperative period (17).
Although they have minimal side effects they do cause patient discomfort and increase cost $(20,22)$. Furthermore, ERAS and standardized chest tube management pathways suggest tolerating a pneumothorax in an asymptomatic patient is safe $(23,24)$.

In a retrospective review of 1,550 CXR and the associated prospectively collected clinical data for 176 patients as part of an RCT (17) found that radiographic findings did not change management in patients that did not have an associated clinical finding, such as shortness of breath, chest pain, tachycardia or increasing oxygen requirements (22). Similarly, in a meta-analysis of 3,649 patients, ordering CXR only when clinically indicated reduced the number of CXR per patient by 3.15 without an increase in mortality, intensive care unit stay, or LOS (25).

CXRs can be classified as routine CXR or on-demand CXR. Routine CXRs are ordered automatically in the 
postoperative period without consideration of clinical findings. On-demand CXRs are ordered only when indicated by clinical findings. Although there is no RCT to support the most appropriate use of CXR, retrospective data suggests on-demand CXRs are a more efficient use of resources. It is reasonable to order a routine CXR in the immediate postoperative period as a baseline acknowledging there is a low probability that it will change management in the absence of associated clinical findings. However, this baseline CXR may be useful if on-demand CXR are ordered later in the postoperative period to confirm a clinical suspicion.

\section{Management}

Once a true alveolar-pleural fistula has been established, management is focused on reducing the duration of the chest tube and/or expediting discharge. Factors such as the number of chest drains (26-32) and use of applied external suction $(33-40)$ have been discussed in the literature. Invasive maneuvers to promote resolution of the air leak such as pleurodesis, placement of bronchial valves and reoperation have been considered. And, finally the role of discharge with a portable device to management a PAL has been supported with data.

\section{Number of chest drains}

After anatomic pulmonary resection thoracic surgeons traditionally leave an apical chest tube to drain air and a basilar chest tube to drain liquid. This tradition has recently been challenged in the literature. There have been four RCTs (26-29), one non-RCT (30), and two metaanalyses $(31,32)$. All studies report outcomes after anatomical pulmonary resections (lobectomy and bilobectomy). Similar to other studies evaluating management of post-operative chest drains, these studies report: duration of chest tube drainage, LOS, postoperative pain and complications.

No study has reported a benefit favoring two chest drains over a single chest drain. A shorter duration of chest drainage and LOS was reported in one study (27) and both meta-analysis $(31,32)$. Three trials $(26,28,30)$ and the one meta-analysis (31) reported decreased postoperative pain, while one study found no difference (29) and one metaanalysis did not analysis postoperative pain (32). The three RCTs that reported postoperative complications (26-28) and both meta-analysis $(31,32)$ found no difference in postoperative complications or need for re-insertion of a chest drain.

Based on the literature a single chest drain is adequate and pooled data suggests a single drain shortens duration of chest drain and LOS. In a patient with a symptomatic air leak not controlled with a single drain a second drain may need to be inserted, however the literature has not found a reduction in drain re-insertion when two drains were used (26-28,31,32). ERAS programs have reported successful implementation of using a single chest tube to management air leaks after pulmonary resection (23). Despite the traditional approach to using two drains, a signal chest drain is adequate for management of an air leak.

\section{Applied external suction versus no applied external suction}

Optimal drainage of an air leak has been long debated. To help clarify the European Society of Thoracic Surgeons (ESTS), the American Association for Thoracic Surgery (AATS), the Society of Thoracic Surgeons (STS), and the General Thoracic Surgery Club (GTSC) have standardized terminology: "applied external suction" is defined as application of negative pressure to the pleural space and "no applied external suction" is defined as the absence of applied negative pressure to the pleural space (41).

Two physiologic mechanisms have been proposed to debate the use of applied external suction to help an air leak heal. The first suggests that external suction promotes airflow through the fistula preventing it from healing and therefore an air leak will heal faster without applied external suction. A contradictory mechanism suggests that suction promotes apposition between the parietal and visceral pleural and thus promotes sealing of the air leak.

Prior to the mainstream development of ERAS, seven RCTs and three meta-analyses were conducted in an effort to determine the optimal use of applied external suction. Interestingly, the primary outcomes of these studies predominantly include parameters important to ERAS programs such as: incidence of PAL, duration of chest tube, LOS, and presence of a residual pneumothorax after chest tube removal.

Two RCTs and one meta-analysis report shorter duration of air leak and chest drain with no applied external suction (33-35) and this translated into a shorter LOS in the meta-analysis (35). Looking at the same outcomes three RCTs and two meta-analyses report no difference with or without applied external suction (36-40,42). One RCT compared alternating applied external suction and 
no applied external on a scheduled basis to continuous no applied external suction, finding a benefit to the alternating regimen (40). A higher rate of residual pneumothorax was found in one RCT supporting no applied external suction which was also reported in one meta-analysis $(37,39)$. Aside from the RCT using an alternating regimen (42), no trial has favored the used of applied external suction, but as noted above five studies have found no difference.

There are several possible explanations for discrepancies in the outcomes: (I) inconsistent protocols between studies may yield different results, especially when the true difference may be small; (II) inconsistent assessments of air leaks by the clinical teams which has been previously shown in the literature (7); (III) and/or application of negative pressure to the pleural space does not significantly impact the time it takes for an alveolar-pleural fistula to heal. Acknowledging the controversies, two programs aimed at standardizing air leak management after pulmonary resection have set a regimen of placing chest drains to applied external suction until POD 1, then moving to no applied external suction unless there is an adverse event $(23,24)$. With consistent protocolized care offered by ERAS programs and more objective assessment of air leaks offered by digital drains, large multi-center RCT may help establish the best regimen for the use of applied external suction in the post-operative period. For now, a consistent approach at an institutional level is likely more important than prolonged debates regarding the benefits and harms of applied external suction.

\section{Invasive maneuvers}

In general the more recent literature has focused on conservative management of air leaks including tolerating pneumothorax on CXR and early discharge with a persistent air leak $(24,43)$. However, invasive maneuvers may be required for patients unable to tolerate an air leak. Furthermore, new and old techniques should be continually explored to find methods to reduce the duration of air leaks and chest tube drainage.

\section{Pleurodesis}

Pleurodesis can be achieved non-operatively at the bedside with a chemical substance or autologous blood. The use of both modalities have been reported in a small cohort studies and RCTs. The literature suggests autologous blood patch pleurodesis (ABPP) may show promise in expediting the resolution of PAL.

Many agents such as talc, silver nitrate, doxycycline, tetracycline bleomycin, and interferon, have been put into the pleural space to induce inflammation and sclerosis leading to adhesions. These agents require opposition between the visceral and parietal pleura to achieve formation of adhesions. The inflammatory response does result in side effects including fever, dyspnea, pain and acute respiratory distress syndrome (ARDS). The literature supporting the use of chemical pleurodesis in for postoperative PAL is limited $(44,45)$. However, a retrospective review of 41 patients after pulmonary resection were treated with chemical pleurodesis with talc, doxycycline, minocycline and a combination of these agents revealed success in 40 patients $(97.6 \%)$. The mean duration of air leak after administration of a sclerosing agent was 2.8 days. One patient (2.5\%) developed empyema (44). Despite these promising results, surgeons may be reluctant to use chemical pleurodesis after pulmonary resection because talc is permanent foreign body with the potential to become infected especially in the setting of a communication with the atmosphere via the alveolar-pleural fistula. While other forms of chemical pleurodesis do not leave a foreign body they are associated with intense pain.

In contrast autologous blood patch as a form of pleurodesis has been more thoroughly studied and has received more discussion in the recent literature. There have been eight small cohort studies with between 11 and 32 patients who were treated with an autologous blood patch for spontaneous and persistent postoperative air leaks. All report successful resolution of the air leaks (46-54). There have been three randomized trials all showing rapid resolution of air leaks with ABPP. One RCT randomized 20 patients with a persistent air leak on the fifth postoperative day to blood patch versus conservative management. The median time for the air leak to seal was 1 day in the blood patch group and 3 days in the conservative management group. This translated into statistically significant shortened duration of chest tube drainage and LOS (55). Another trial randomized 25 patients to 50 or $100 \mathrm{~mL}$ of instilled blood or conservative management. This study reported a mean of 2.3 and 1.5 days for resolution of the air leak for the groups receiving 50 and $100 \mathrm{~mL}$ of blood, respectively. This was significantly shorter than a mean of 6.3 days in the group treated with conservative management. There were no infectious complications (56). The utility of blood patches for the treatment of persistent air leak has been the subject of two systematic reviews $(45,56)$. Both reviews 
include persistent air leaks resulting from spontaneous pneumothorax and post pulmonary resection. Both reviews concluded $\mathrm{ABPP}$ has promising results with minimal complications include cost, and therefore it should consider for patients with persistent air leaks, yet more research is needed $(45,57)$.

To perform ABPP, typically 50 to $120 \mathrm{~mL}$ of blood is drawn from a peripheral site and injected into the chest tube. There has been some controversy around the quantity of blood to use (45). One RCT did compare 50 or $100 \mathrm{~mL}$ and did not find a statistically significant difference in the time for resolution of air leak (55). Air leaks are typically reported to resolve within 12 to 24 hours (57). It is important to use meticulous sterile technique because blood is a known culture medium for bacteria (56).

The literature suggests that ABPP is an effective means of resolving air leaks. The data does include a mix of spontaneous pneumothorax and postoperative patients. The complication rate is reported to be relatively low. Given the available evidence ABPP should be considered for treatment of air leak as part of an ERAS program. As ERAS programs evolve more trials should be designed to compare ABPP to discharge with a portable device looking at duration of chest drain, LOS and patient experience as primary outcomes.

\section{Bronchial valves}

Endobronchial Valve for Emphysema Palliation Trial (VENT) compared bronchial valves to standard medical management in the treatment of heterogeneous emphysema using a RCT. The VENT study found modest improvement in pulmonary function, exercise tolerance and symptoms favoring bronchial valves at the risk of increased exacerbations of COPD, pneumonia, and hemoptysis (58). Bronchial valves are currently marketed as endobronchial valves (Zephyr, PulmonX Inc.) and intrabronchial valves (IBV/SVS system, Spiration Inc.) (59). Implantation of a bronchial valves is well described and can be divided into three steps: (I) identification of the segment or subsegment of the bronchial tree leading to the air leak using serial balloon inflations with an indwelling chest drain and observation of resolution of the air leak, (II) choosing the appropriate valve size (using sizers provided by the manufacturers) and (III) deployment of the valve $(60,61)$. At present endobronchial valves do not meet US Food and Drug Administration-approved criteria to treat air leaks however they have been used through the Humanitarian Device Exemption (61).
Although there is recent literature it has not focus on the postoperative air leak. Data for use for the treatment of air leaks has been limited to case series of which few studies have included postoperative air leaks (59). And, no series has been restricted to postoperative air leaks alone with all including patients with air leaks after spontaneous and non-spontaneous (trauma and iatrogenic) pneumothorax in addition to postoperative air leaks. An international case series of 40 patients who had bronchial valves placed for air leaks included eight patients who were postoperative air leaks. In this study, after placement of a bronchial valve 19 of the 40 patients $(47.5 \%)$ had complete resolution of their air leak, 18 of 40 patients (45\%) had improvement of their air leak, two patients (5\%) had no response and one patient $(2.5 \%)$ was not reported. The median and mean duration of chest drainage after the procedure was 7.5 days and 21 days, respectively. The median and mean LOS after valve placement was 19 days and 11 days, respectively (62). In another series nine patients with a median air leak of greater than 4 weeks, had attempted treatment with a bronchial valve. Seven patients $(77.8 \%)$ had successful placement of a valve requiring eight procedures. A median of 3.5 valves were used. The median duration of air leak after valve placement was 1 day and four patients were discharged within 2 to 3 days of valve placement (63). In another study, 21 patients with air leaks had 24 procedures to place bronchial valves. Ten patients were post pulmonary resection. Patients required a chest drain for median of 15 days and had a median LOS of 5 days after valve placement (64).

The use of bronchial valves for postoperative air leaks is limited to small case series which include nonoperative and postoperative patients. The success in these series has been limited. That said, these devices have mainly been used as a last resort in patient who have failed other management options. It is possible that earlier use may expedite discharge. A multicenter prospective RCT, valves against standard therapy (VAST), to compare bronchial valves to standard management of air leaks is currently enrolling $(59,65)$. This study will not be limited to postoperative air leaks. Until more data is available bronchial valves should remain a last resort for management of postoperative air leaks. In addition to the complications from the VENT study noted above, placement of bronchial valves for postoperative leaks should also include treatment failure and atelectasis.

\section{Re-operation}

There are no trials comparing re-operation to other methods 
of managing postoperative air leaks. There are multiple intraoperative maneuvers described to prevent air leaks including: buttressing the staple line, use of topical sealants, pleural tent, and induction of pneumoperitoneum (66). However, these have not been evaluated in the setting of re-operation.

That said, re-operation is rarely required (67). It is likely to be most helpful when a large air leak is unexpected identified within the first 24 hours after pulmonary resection. In this setting early reoperation can rule out $\mathrm{BPF}$, identify and staple off an injury to the parenchyma or redo the staple lines applying one of the maneuvers mentioned above.

ERAS protocols for pulmonary resection have not included re-operation but tend to focus on more conservative management of air leaks (23). Similar to bronchial valves, re-operation should be limited to few patients and selectively used in the very early postoperative period.

\section{Outpatient management}

Irrespectively of best practices and ERAS protocols, PAL will occur. To get the most benefit of ERAS programs and until invasive maneuvers are better explored, protocols should include outpatient management of PAL. These protocols need to define three points: (I) when to connect a patient to a portable device, (II) when and how to follow up as an outpatient, and (III) criteria to remove the chest drain.

There have been five studies that show air leaks can be safely managed in the postoperative period with portable devices. In a retrospective study of 45 patients post pulmonary resection with a PAL, 44 patients $(98 \%)$ were successfully management on an outpatient basis (68). And, 18 patients were all managed without a failure in another study (69). In another study, 55 patients with an air leak were placed on a Heimlich valve of which 22 had resolution within a day, but 33 patients had a persistent air leak. Six of these patients failed the Heimlich valve requiring drainage via a water seal or applied external suction. All of these patients had an air leak of five or more using the Robert David Cerfolio Classification System. Ultimately all 33 patients were discharged home with a Heimlich valve (2). Similarly, 32 of 36 patients (89\%) were successfully managed with a portable device in another retrospective study (70). A larger study of 193 patients revealed that 190 were managed without a significant complication. The three patients that did have complications were all immunosuppressed (21). A retrospective review of prospectively collected data over
5 years of 65 patients discharged with a portable device found reduced LOS (mean 3.65 days) compared to the Society of Thoracic Surgeons (STS) database as a control (mean 6.2 days). There were two thromboembolic events in the study (71). Another retrospective review of 73 patients discharged over a 10 -year period again showed a reduced LOS (mean 3.88 days) compared to a control arm at the same institution (mean 5.68 days). There was no increase in complication rate for patients discharged with a portable drain and only two readmissions. This translated into a saving of 145 patient days in hospital and $\$ 686.72$ per patient (72).

In another study, patients were connected to a Heimlich valve on POD 4 and discharged between POD 5 and 11. Patients were instructed how to check for an air leak, once it resolved the tube was removed. If the air leak exceeded 2 weeks the patient was admitted for a trial of chest tube clamping prior to removal of the chest drain (2). Later the same author reported connecting 193 patients to a portable device on POD 3 and discharging on POD 4. All patients were discharged with oral antibiotics. All chest drains were removed at a median of 16.5 days after discharge even in the setting of an air leak or pneumothorax on CXR (21). In another study, patients were evaluated within 3 days of discharge and CXR were obtained at all follow up visits. Chest drains were removed at a mean of 4.7 days after discharge (71). In another study, patients were followed up 4 to 5 days after discharge with a CXR and assessment for an air leak. Chest drains were removed with a mean of 8.3 days after discharge (72).

In summary, there is retrospective evidence that patients can be safely discharged home with a portable device. Studies have discharged patients between POD 4 and 5 with follow up within 3 to 5 days. Chest drains can typically be removed within 4 to 11 days after discharge and one study shows all chest drains can be removed roughly 17 days after discharge even in the presence of an air leak or pneumothorax. Future efforts will promote early identification of patients expected to require discharge with a portable device and possibly prediction models to estimate the day that the air leak will resolve. This will facilitate earlier discharge with a portable device and scheduling of follow up reducing health care costs.

\section{Conclusions}

In the management of postoperative air leaks, digital drains are expected to benefit ERAS programs by providing 
objective, documented evidence of a resolution of an air leak. The use of routine CXR can be minimized unless clinically indicated. There is no clear evidence that the use of applied external suction promotes or prevents air leaks, and therefore consist practices at an institutional level will provide the best chest tube management. The most promising invasive maneuver to promote early resolution of an air leak is ABPP. However, until the role of ABPP is defined with a larger RCT, conservative management with early discharge with a portable device will be required for some patients with a PAL.

Postoperative air leaks are expected to remain the most common problems in thoracic surgery. Recent advances in technology and evidence-based practices in thoracic surgery provide a solid base for the development of protocols within ERAS programs to management air leaks. And, ERAS programs will provide a platform to better assess technology and controversies to advance postoperative care for thoracic surgery patients.

\section{Acknowledgements}

None.

\section{Footnote}

Conflicts of Interest: The authors have no conflicts of interest to declare.

\section{References}

1. Rice TW, Kirby TTJ. Prolonged air leak. Chest Surg Clin N Am 1992;2:803-11.

2. Cerfolio RJ, Bass CS, Pask AH, et al. Predictors and treatment of persistent air leaks. Ann Thorac Surg 2002;73:1727-30; discussion 1730-1.

3. Antanavicius G, Lamb J, Papasavas P et al. Initial chest tube management after pulmonary resection. Am Surg 2005;71:416-9.

4. Brunelli A, Monteverde M, Borri A, et al. Predictors of prolonged air leak after pulmonary lobectomy. Ann Thorac Surg 2004;77:1205-10.

5. Brunelli A, Cassivi SD, Halgren L. Risk factors for prolonged air leak after pulmonary resection. Thorac Surg Clin 2010;20:359-64.

6. Cerfolio RJ. Advances in thoracostomy tube management. Surg Clin North Am 2002;82:833-48.

7. McGuire AL, Petrich W, Maziak DE, et al. Digital versus analogue pleural drainage phase 1: prospective evaluation of interobserver reliability in the assessment of pulmonary air leaks. Interact Cardiovasc Thorac Surg 2015;21:403-7.

8. Varela G, Jimenez MF, Novoa NM, et al. Postoperative chest tube management: measuring air leak using an electronic device decreases variability in the clinical practice. Eur J Cardiothorac Surg 2009;35:28-31.

9. Cerfolio RJ, Bryant AS. The benefits of continuous and digital air leak assessment after elective pulmonary resection: a prospective study. Ann Thorac Surg 2008;86:396-401.

10. Brunelli A, Salati M, Refai M, et al. Evaluation of a new chest tube removal protocol using digital air leak monitoring after lobectomy: a prospective randomised trial. Eur J Cardiothorac Surg 2010;37:56-60.

11. Filosso PL, Ruffini E, Solidoro P, et al. Digital air leak monitoringafter lobectomy for primary lung cancer in patients with moderate COPD: Does a fast-tracking algorithm reduce postoperative costs and complications? J Cardiovasc Surg 2010;51:1-5.

12. Bertolaccini L, Rizzardi G, Filice MJ, et al. 'Six sigma approach'-an objective strategy in digital assessment of postoperative air leaks: a prospective randomised study. Eur J Cardiothorac Surg 2011;39:e128-32.

13. Pompili C, Detterbeck F, Papagiannopoulos K, et al. Multicenter international randomized comparison of objective and subjective outcomes between electronic and traditional chest drainage systems. Ann Thorac Surg 2014;98:490-6; discussion 496-7.

14. Filosso PL, Nigra VA, Lanza G, et al. Digital versus traditional air leak evaluation after elective pulmonary resection: a prospective and comparative monoinstitutional study. J Thorac Dis 2015;7:1719-24.

15. Cho HM, Hong YJ, Byun CS, et al. The usefulness of WiFi based digital chest drainage system in the post-operative care of pneumothorax. J Thorac Dis 2016;8:396-402.

16. Lijkendijk M, Licht PB, Neckelmann K. Electronic versus traditional chest tube drainage following lobectomy: a randomized trial. Eur J Cardiothorac Surg 2015;48:893-8.

17. Gilbert S, McGuire AL, Maghera S, et al. Randomized trial of digital versus analog pleural drainage in patients with or without a pulmonary air leak after lung resection. J Thorac Cardiovasc Surg 2015;150:1243-9.

18. Plourde M, Jad A, Dorn P, et al. Digital Air Leak Monitoring for Lung Resection patients: A Randomized Controlled Clinical Trial. Ann Thorac Surg 2018. [Epub ahead of print].

19. Varela G, Jimenez MF, Novoa N. Portable chest drainage 
systems and outpatient chest tube management. Thorac Surg Clin 2010;20:421-6.

20. Cerfolio RJ, Bryant AS. The management of chest tubes after pulmonary resection. Thorac Surg Clin 2010;20:399-405.

21. Cerfolio RJ, Minnich DJ, Bryant AS. The removal of chest tubes despite an air leak or a pneumothorax. Ann Thorac Surg 2009;87:1690-4; discussion 1694-6.

22. French DG, Dilena M, LaPlante S, et al. Optimizing postoperative care protocols in thoracic surgery: best evidence and new technology. J Thorac Dis 2016;8:S3-11.

23. Gonfiotti A, Viggiano D, Voltolini L, et al. Enhanced recovery after surgery and video-assisted thoracic surgery lobectomy: the Italian VATS Group surgical protocol. J Thorac Dis 2018;10:S564-70.

24. Drahush N, Miller AD, Smith JS, et al. Standardized Approach to Prolonged Air Leak Reduction After Pulmonary Resection. Ann Thorac Surg 2016;101:2097-101.

25. Reeb J, Falcoz PE, Olland A, et al. Are daily routine chest radiographs necessary after pulmonary surgery in adult patients? Interact Cardiovasc Thorac Surg 2013;17:995-8.

26. Gómez-Caro A, Roca MJ, Torres J, et al. Successful use of a single chest drain postlobectomy instead of two classical drains: a randomized study. Eur J Cardiothorac Surg 2006;29:562-6.

27. Pawelczyk K, Marciniak M, Kacprzak G, et al. One or two drains after lobectomy? A comparison of both methods in the immediate postoperative period. Thorac Cardiovasc Surg 2007;5 5:313-6.

28. Okur E, Baysungur V, Tezel C, et al. Comparison of the single or double chest tube applications after pulmonary lobectomies. Eur J Cardiothorac Surg 2009;35:32-5.

29. Tanaka M, Sagawa M, Usuda K, et al. Postoperative drainage with one chest tube is appropriate for pulmonary lobectomy: a randomized trial. Tohoku J Exp Med 2014;232:55-61.

30. Alex J, Ansari J, Bahalkar P, et al. Comparison of the immediate postoperative outcome of using the conventional two drains versus a single drain after lobectomy. Ann Thorac Surg 2003;76:1046-9.

31. Zhou D, Deng XF, Liu QX, et al. Single chest tube drainage is superior to double chest tube drainage after lobectomy: a meta-analysis. J Cardiothorac Surg 2016;11:88.

32. Zhang X, Lv D, Li M, et al. The single chest tube versus double chest tube application after pulmonary lobectomy: A systematic review and meta-analysis. J Cancer Res Ther
2016;12:C309-16.

33. Marshall MB, Deeb ME, Bleier JI, et al. Suction vs water seal after pulmonary resection: a randomized prospective study. Chest 2002;121:831-5.

34. Cerfolio RJ, Bass C, Katholi CR. Prospective randomized trial compares suction versus water seal for air leaks. Ann Thorac Surg 2001;71:1613-7.

35. Lang P, Manickavasagar M, Burdett C, et al. Suction on chest drains following lung resection: evidence and practice are not aligned. Eur J Cardiothorac Surg 2016;49:611-6.

36. Alphonso N, Tan C, Utley M, et al. A prospective randomized controlled trial of suction versus non-suction to the under-water seal drains following lung resection. Eur J Cardiothorac Surg 2005;27:391-4.

37. Prokakis C, Koletsis EN, Apostolakis E, et al. Routine suction of intercostal drains is not necessary after lobectomy: a prospective randomized trial. World J Surg 2008;32:2336-42.

38. Brunelli A, Monteverde M, Borri A, et al. Comparison of water seal and suction after pulmonary lobectomy: a prospective, randomized trial. Ann Thorac Surg 2004;77:1932-7.

39. Coughlin SM, Emmerton-Coughlin H, Malthaner R. Management of chest tubes after pulmonary resection: a systematic review and meta-analysis. Can J Surg 2012;55:264-70.

40. Deng B, Tan QY, Zhao YP, et al. Suction or non-suction to the underwater seal drains following pulmonary operation: meta-analysis of randomized controlled trials. Eur J Cardiothorac Surg 2010;38:210-5.

41. Brunelli A, Beretta E, Cassivi SD, et al. Consensus definitions to promote an evidence-based approach to management of the pleural space. A collaborative proposal by ESTS, AATS, STS, and GTSC. Eur J Cardiothorac Surg 2011;40:291-7.

42. Brunelli A, Sabbatini A, Xiume F, et al. Alternate suction reduces prolonged air leak after pulmonary lobectomy: A Randomized Comparison Versus Water Seal, Ann Thorac Surg 2005;80:1052-5.

43. Lackey A, Mitchell JD. The cost of air leak: physicians' and patients' perspectives. Thorac Surg Clin 2010;20:407-11.

44. Liberman M, Muzikansky A, Wright CD, et al. Incidence and risk factors of persistent air leak after major pulmonary resection and use of chemical pleurodesis. Ann Thorac Surg 2010;89:891-7.

45. Manley K, Coonar A, Wells F, et al. Blood patch for persistent air leak: a review of the current literature. Curr Opin Pulm Med 2012;18:333-8. 
46. Robinson CL. Autologous blood for pleurodesis in recurrent and chronic spontaneous pneumothorax. Can J Surg 1987;30:428-9.

47. Cagirici U, Sahin B, Cakan A, et al. Autologous blood patch pleurodesis in spontaneous pneumothorax with persistent air leak. Scand Cardiovasc J 1998;32:75-8.

48. Ando M, Yamamoto M, Kitagawa C, et al. Autologous blood-patch pleurodesis for secondary spontaneous pneumothorax with persistent air leak. Respir Med 1999;93:432-4.

49. Rivas de Andrés JJ, Blanco S, de la Torre M. Postsurgical pleurodesis with autologous blood in patients with persistent air leak. Ann Thorac Surg 2000;70:270-2.

50. Lang-Lazdunski L, Coonar AS. A prospective study of autologous ‘blood patch' pleurodesis for persistent air leak after pulmonary resection. Eur J Cardiothorac Surg 2004;26:897-900.

51. Ozpolat B. Autologous blood patch pleurodesis in the management of prolonged air leak. Thorac Cardiovasc Surg 2010;58:52-4.

52. Droghetti A, Schiavini A, Muriana P et al. Autologous blood patch in persistent air leaks after pulmonary resection. J Thorac Cardiovasc Surg 2006;132:556-9.

53. Athanassiadi K, Bagaev E, Haverich A. Autologous blood pleurodesis for persistent air leak. Thorac Cardiovasc Surg 2009;57:476-9.

54. Oliveira FH, Cataneo DC, Ruiz RL Jr, et al. Persistent pleuropulmonary air leak treated with autologous blood: results from a university hospital and review of literature. Respiration 2010;79:302-6.

55. Shackcloth MJ, Poullis M, Jackson M, et al. Intrapleural instillation of autologous blood in the treatment of prolonged air leak after lobectomy: a prospective randomized controlled trial. Ann Thorac Surg 2006;82:1052-6.

56. Andreetti C, Venuta F, Anile M, et al. Pleurodesis with an autologous blood patch to prevent persistent air leaks after lobectomy. J Thorac Cardiovasc Surg 2007;133:759-62.

57. Chambers A, Routledge T, Billè A, et al. Is blood pleurodesis effective for determining the cessation of persistent air leak? Interact Cardiovasc Thorac Surg 2010;11:468-72.

58. Sciurba FC, Ernst A, Herth FJ, et al; VENT Study Research Group. A randomized study of endobronchial valves for advanced emphysema. N Engl J Med 2010;363:1233-44.

59. Dugan KC, Laxmanan B, Murgu S, et al. Management of
Persistent Air Leaks. Chest 2017;152:417-23.

60. Mahajan AK, Doeing DC, Hogarth DK. Isolation of persistent air leaks and placement of intrabronchial valves. J Thorac Cardiovasc Surg 2013;145:626-30.

61. Kovitz KL, French KD. Endobronchial valve placement and balloon occlusion for persistent air leak: procedure overview and new current procedural terminology codes for 2013. Chest 2013;144:661-5.

62. Travaline JM, McKenna RJ Jr, De Giacomo T, et al; Endobronchial Valve for Persistent Air Leak Group. Treatment of persistent pulmonary air leaks using endobronchial valves. Chest 2009;136:355-60.

63. Gillespie CT, Sterman DH, Cerfolio RJ, et al. Endobronchial valve treatment for prolonged air leaks of the lung: a case series. Ann Thorac Surg 2011;91:270-3.

64. Reed MF, Gilbert CR, Taylor MD, et al. Endobronchial Valves for Challenging Air Leaks. Ann Thorac Surg 2015;100:1181-6.

65. Mahajan AK, Khandhar SJ. Bronchoscopic valves for prolonged air leak: current status and technique. J Thorac Dis 2017;9:S110-5.

66. Merritt RE, Singhal S, Shrager JB. Evidence-based suggestions for management of air leaks. Thorac Surg Clin 2010;20:435-48.

67. Burt BM, Shrager JB. Prevention and management of postoperative air leaks. Ann Cardiothorac Surg 2014;3:216-8.

68. Ponn RB, Silverman HJ, Federico JA. Outpatient chest tube management. Ann Thorac Surg 1997;64:1437-40.

69. Lodi R, Stefani A. A new portable chest drainage device. Ann Thorac Surg 2000;69:998-1001.

70. Rieger KM, Wroblewski HA, Brooks JA, et al. Postoperative outpatient chest tube management: initial experience with a new portable system. Ann Thorac Surg 2007;84:630-2.

71. Royer AM, Smith JS, Miller A, et al. Safety of outpatient chest tube management of air leaks after pulmonary resection. Am Surg 2015;81:760-3.

72. Schmocker RK, Vanness DJ, Macke RA, et al. Outpatient air leak management after lobectomy: a CMS cost analysis. J Surg Res 2016;203:390-7.

Cite this article as: French DG, Plourde $M$, Henteleff H, Mujoomdar A, Bethune D. Optimal management of postoperative parenchymal air leaks. J Thorac Dis 2018;10(Suppl 32):S3789-S3798. doi: 10.21037/jtd.2018.10.05 